\title{
Sur l'évolution de la fonction respiratoire chez les œufs d'Amphibiens.
}

\author{
Par
}

E. Bataillon.

Eingegangen am 29. April 1901.

E. Godlewski jun. vient de publier dans ce Recueil (Archiv f. Entwickelungsmechanik. Bd. XI. Heft 2/3) d'intéressantes recherches sur les échanges gazeux des oufs de Rana temporaria aux premiers stades du développement. Ses résultats confirment d'une façon remarquable ceux que j'ai moi-même enregistrés en $1896^{1}$, et 1897 2). GoDLEwski n'a certainement pas eu connaissance de ces travaux; et, si je les rappelle, c'est qu'au point de vue de la mécanique du développement, ils ont une portée beaucoup plus considérable que lessiens je ne vise que la $2^{\text {me }}$ partie: déterminations quantitatives:

Mon objectif était d'établir une courbe continue de l'activité respiratoire. J'ai cherché d'abord a apprécier l'élimination de l'acide carbonique par la méthode qu'il indique pag. 607 et 608 (pag. 305 de mon mémoire). Cette méthode m'a donné des jalons fondamentaux qui demandaient à être rattachés.

Par un procédé identique à celui qu'il décrit pag. 605 pag. 306 de mon mémoire), je me suis assuré que l'absorption d'oxygène montre des oscillations de même ordre.

J'ai fait ressortir l'insuffisance de tonte cette techmique et me suis arrêté, pour établir une courbe régulière de l'élimination d'acide carbonique, à un procédé beancoup plus simple et qui mérite d'être pris en considération par la précision de ses données. SJ'ai commencé par m'assurer que, dans une eau contenant une faible quantité de baryte, les œufs d'Amphibiens, comme ceux de 'Téléostéens, évoluent très bien et normalement. Le fait acquis, je plaçai dans une quantité déterminée de liquide titré et rougi à la Phtaléine, une masse d'œufs connue; le temps nécessaire au virage me donnait la mesure de l'activité respiratoire.« Bataillon, loc. cit. (2) pag. 306.

1) E. Bataillon, Evolution de la fonction respiratoire chez les embryons d'Amphibiens et de Téléostéens (C. R. et Mém. de la Soc. de Biologie. 1896.

?) E. Batallion, Nouvelles recherches sur les mécanismes de l'évolution. Les premiers stades du développement chez les Amphibiens et les Poissons (Arch. de Zool. Exple. 1897. 
Sur l'évolution de la fonction respiratoire chez les cufs d'Amphibiens. 303

Depuis que j'ai inauguré cette méthode, elle a été employée avec avantage par d'autres biologistes. GodLewskr était done mal informé lorsqu'il a écrit: -Dieses Gebiet der quantitativen Untersuchungen des Athmungsprocesses, wälrend der Entwickelung, ist bis jetzt nur wenig in Angriff genommen worden desswegen stand mir anch $z u$ diesem Zwecke keine in der Entwickelungsmechanik angewandte Methode zur Verfügung. GodLewski, loc. cit. pag. 603.

Il reconnaîtra certainement que ma méthode sdes virages vant quelque chose si je fais ressortir une identité parfaite entre ses résultats et les miens.

Dans quelles limites varie l'élimination d'acide carbonique quand il dose avec l'appareil de Bonnier et Mangln?

Pour 100 oufs et pour $24 \mathrm{~h}$, entre 0,0995 et 1,611 . Or, mes expériences m'ont donné 0,08 et 1,44 . Il est difficile d'exiger une coneordance plus rigoureuse.

Quant à l'évolution de la fonction au point de vue quantitatif, GoDLEwskI a raison de ne pas considérer ses résultats comme définitifs.

Il est bien exact que, d'une façon très-générale, l'activité respiratoire croît avec la marche du développement. Mais, avec des opérations trop espacées, on laisse dans l'ombre des oscillations du plus haut intérêt.

J'en ai signalé une au début de ce qu'on appelle la période du revêtement ectodermique. Lorsque l'émiettement des blastomères au pôle animal atteint une certaine limite, l'extension ou le remaniement au pôle végétatif semble se buter a un obstacle physiologique. La courbe tombe assez brusquement pour se relever plus lentement jusqu'à l'occlusion du blastopore.

Ici, j'enregistre une nouvelle baisse qui peut s'expliquer par une difficulté mécanique de même ordre.

Je suis très heureux de découvrir dans les tableaux de GodLEwski la marque de cette 2me oscillation. Dans ses mesures de l'absorption d'oxygène par la dépression (Tabl. III et IV) les chiffres correspondant i la réduction de volume sont les suivants:

$$
\begin{aligned}
& \text { III: } 1,04 ; 0,92 ; 2,30 ; \\
& \text { IV: } 2,57 ; 2,76 ; 1,42 ; 4,47 .
\end{aligned}
$$

Dans la $2^{\text {e }}$ expérience, la chute correspond au $3^{\text {ine }}$ jour, tandis que dans la 1ère, elle répond au second et au début du troisième.

C'est qu'il faut tenir compte de la température. La date de l'occlusion du blastopore dans le $2^{\text {ème }}$ cas indiquerait une t. moyenne d'environ $15^{\circ}$ (c'est en effet la moyenne des chiffres fournis). Dans le 1er cas, la température aurait été plus élevée. Or, nous trouvons là précisement, au moment du premier dosage $19,2^{\circ}$, chiffre qui n'apparaît nulle part dans l'opération suivante. Vers $18^{\circ}$ ou $19^{\circ}$ c'est bien le $2^{\text {me }}$ jour que le blastopore se ferme.

Ainsi, malgré des dosages trop espacés, le deuxième accident de ma conrbe, celui qui, au point de vue du temps, se montre le plus dilaté, se retrouve dans les expériences de GodLewski.

Je ne puis donc voir dans ces résultats incomplets qu'une confirmation remarquable des miens.

Et ces derniers demandaient à etre rappelés pour d'autres raisons:

10. Il était bon de signaler à l'attention des biologistes une méthode d'une application facile, mais pré cieuse surtout par la précision des résultats qu'elle donne. 
304 E. Bataillon, Sur l'évolution de la fonction respiratoire chez les oufs d'Auph.

$2^{\circ}$. Si, comme j'en ai la conviction, une courbe de l'activité respiratoire offre quelque intérêt au point de vue dela mécanique du développement, cet intérêt devient surtout manifeste quand on peut rapporter commejel'ai faitlesoscillations fonctionnelles à des étapes caractérisées de l'évolution.

3o. Enfin, ces variations sont particuliérement suggestives quand je les souligne dans le groupe des Poissons pour des stades embryologiques comparables.

Université de Dijon (France). 